\title{
Additional Doses of Vaccines against COVID-19-Attempting to Enhance the Immune Response
}

\author{
Fernando Álvez* \\ Pediatrician, Senior Consultant Group on Genetics, Vaccines and Pediatric Infections (GENVIP). Infectious Diseases \\ and Vaccines Unit (UNIV). University Clinical Hospital. Santiago de Compostela, Spain. Spanish Society of Pediatric \\ Infectology
}

\author{
Keywords \\ Coronavirus, SARS-CoV-2, COVID-19, COVID-19 vaccines, Additional vaccination doses, Response immune, Antibody- \\ dependent enhancement
}

\begin{abstract}
Abbreviations
Nab: Neutralizing Antibodies; VOC: Variants of Concern; ADE: Antibody-Dependent Enhancement; RBD: Receptor Binding Domain; NTD: N-Terminal Domain
\end{abstract}

\section{Introduction}

Vaccination against COVID-19 with a complete primary series is essential. To date, COVID-19 vaccines have demonstrated high vaccine effectiveness against severe disease, hospitalization and death from COVID-19 in the majority of the properly vaccinated population. No vaccine including the COVID-19 vaccines is $100 \%$ effective so some breakthrough infections are possible. Moreover, evidence has been emerging of a "weakening" of immunity after passing the infection as well as a decrease in the effectiveness of the vaccines that have been administered against this infection. For this reason, according to many opinions, it will be necessary to reinforce the immune system through additional doses of the available vaccines. The general purpose of these doses is to restore the protection that has declined over time to an insufficient level in persons who initially responded adequately or less than expected, after a complete series of primary vaccination. Currently, there is some scientific debate regarding whether an additional dose of COVID-19 vaccines really constitutes a suitable booster, especially in the nonelderly population without other underlying risk factors or associated pathologies. It is important to assess the existing scientific contribution to the best way to manage these vaccines and to examine the key differences between primary immunizations able to generate a robust immune memory, versus a booster that potentiates the memory response when the initial protection has really declined.

With this decision made and although it may seem the same thing, it could be necessary to distinguish between the administration of a booster dose for persons with a normal immune system in whom the benefits are more difficult to appreciate, and an extra dose for persons with weakened immune systems or carriers of underlying pathologies in whom such doses will be very important.

Thus, could vaccines provide a true "sterilizing immunity" and primary immunization be sufficient in people without risk factors?

\section{Possible Weakening of the Immune System against Severe Acute Respiratory Coronavirus 2 (SARS-CoV-2)}

After the infection has cleared and logically after starting and implanting immunization in the general population with the available vaccines, the expectation is that the humoral immunity of antibodies achieved would ideally be sufficient and also long-lasting. Considering what happens after the infection has passed, the levels of neutralizing antibodies (NAb) generated should be highly indicative of protection

\footnotetext{
*Corresponding author: Pediatrician, Senior Consultant Group on Genetics, Vaccines and Pediatric Infections (GENVIP). Infectious Diseases and Vaccines Unit (UNIV). University Clinical Hospital. Santiago de Compostela, Spain. Spanish Society of Pediatric Infectology, Avenida Figueroa 11-3², 15705 Santiago de Compostela, Spain

Accepted: January 22, 2022

Published online: January 24, 2022

Citation: Álvez F (2022) Additional Doses of Vaccines against COVID-19 - Attempting to Enhance the Immune Response. Vaccine Res Dev 3(1):9-15
} 
against infection and disease. And the same can be assumed with vaccines because of the imprinting of specific antigen characteristics on the host. Although there is evidence of a waning of immunity both after passing SARS-CoV-2 infection and after vaccination, in immunocompetent persons neither their immune systems nor the vaccines are failing. In convalescent persons, the duration of this protective response is not yet well-defined, as discrepant findings have been reported. From an observed trend of the waning of the primary immune responses within a few months [13] to others expressing detectable Nab against SARS-CoV-2 and persisting in most subjects for 6 months or at least 12 months after infection, even in asymptomatic infections [46]. It should be noted that the decrease in time of achieved antibodies is neither unexplained nor alarming. This decline is a common pattern in many infectious diseases and does not necessarily indicate the loss of long-term protective immunity.

\section{Is there a Similar Outcome of Immune Response after Primary Vaccination?}

So far, vaccines against COVID-19 are being effective even considering that, as with other vaccines, NAb titers may decline over time. Several studies of populations vaccinated against SARS-CoV-2 have shown a decrease in antibody levels in the first 6 months and with more attenuated responses against the new variants of concern (VOC) of the SARS-CoV-2 virus, leading to less protection against asymptomatic and mild cases. Fortunately, however, the effectiveness against severe infection and deaths remains relatively long-lasting, even taking into account certain limitations of the studies [711]. At present, there is particular concern about the Delta and Omicron variants most active and transmissible VOC, and its potential immunologic escape, and that the vaccines would not be as effective. However, studies are consistently indicating that vaccines provide sufficient levels of protection against symptomatic and severe disease as well as death caused by Delta variant. [7,8,12-14]. For circulating VOC (as Omicron) that have not yet evolved sufficiently to escape the vaccine-induced immune response, no convincing cut-off point is yet known to indicate what level of antibody-threshold of protection - is most optimal for achieving adequate protection. It is not clear whether a reduction in NAb activity means that vaccines will be ineffective, though it is expected that despite such a reduction, the immune response achieved will still be sufficiently high to confer protection. In short, the available vaccines offer remarkable protection to immunocompetent individuals against SARS-CoV-2 from symptomatic infection, as well as asymptomatic infection and thus transmission. Specifically, this was evidenced by the results of clinical trials and observational studies of mRNA vaccines [15]. And it seems rational that vaccinated persons reduce - not eliminate - the risk of transmission and contagion of the virus to the people in their environment, with a special contribution to those who cannot be vaccinated such as children under 5 years of age or persons with immunosuppression, although there is still insufficient evidence. Studies on SARS-CoV-2 transmission have confirmed that vaccinated persons are less likely to transmit the virus, including the VOCS, to their contacts than unvaccinated persons. Therefore, those susceptible persons who are not immune at all against COVID-19 have a significantly lower risk of infection (and hospitalization) when other members of their close environment are immune, either by natural infection or by being properly vaccinated $[16,17]$. A reduction in viral load has been observed by PCR quantification in individuals immunized with the mRNA BNT162b2 vaccine [18], and in turn, a lower viral load has been associated with a reduction in viral transmission [19]. Vaccination, although it reduces the risk of infection with the Delta variant and hastens viral clearance - as the viral load declines more rapidly in the first few days - is not entirely effective in preventing its transmission in environments with prolonged exposure [20].

\section{What about the Immune Response over Time?}

The available information from systematic testing and sampling studies are reporting a moderate decline in protection (but always referring to the amount of NAb) against infection over time, and that therefore would emerge reinfections or breakthrough infections in those already vaccinated $[9,10]$. However, and taking into account what occurs in convalescent patients once the infection has cleared, the antibody titers still circulating can predict a certain level of protection against symptomatic infection. It should be pointed out that the main objective of SARS-CoV-2 vaccines is to protect against severe disease rather than infection, and thus several welldesigned studies have shown sustained vaccine effectiveness, in some cases beyond 5 months, against severe disease in the majority of adults, especially among persons without significant underlying pathologies $[12,21,22]$. Regarding the estimated reductions in vaccine protection against infection, the data vary widely and are not easy to interpret given the versatility in prevalence, behavior and circulating variants among studies and countries [23]. Their comparison and interpretation is not very reliable to establish a breakdown in the protection against infection over time, and with more significance in the elderly due to the attributed senescence of their capacity to generate antibodies. A study, such as the one carried out in New York (USA) on behavioral changes in the estimated vaccine effectiveness against infection, showed that in the properly immunized, the drop in effectiveness was greater in those $<65$ years of age (from $92-93 \%$ to $72-78 \%$ ) vs. $>65$ years (from $90.5 \%$ to $80 \%$ ) [24].

\section{Requirement for an Extra Dose or Booster Dose of Vaccine}

It is essential to understand how immunity to SARS-CoV-2 evolves along the time in order to plan the course of this pandemic and to make important decisions on its prevention through immunization. The finding of a drop in the amount of NAb after primary immunization and the diversity with new variants, besides promoting mistrust in people about the goodness and benefits of the vaccines, have motivated the convenience of an additional vaccine dose.

The purpose is to prolong protection and to increase its magnitude even more, especially in certain risk groups, but 
with some discrepancies in its suitability for the general immunocompetent population. Thus, the decision was taken in some countries, backed by organizations such as WHO, CDC, FDA, ACIP and ECDC [25-29] to administer an additional dose after a period of time following primary vaccination. A shared reasonable and preferential decision for those persons with associated pathologies and, those with alterations in their immune system in whom adequate protection has not been achieved. Also, extendable to the elderly people due to their expected deterioration in the antibody response and, if the new variants of the virus that are circulating express different antigens capable of motivating inadequate immune responses to vaccines. It is already clear as it is being discussed that from an immunological point of view plasma Nab titers are expected to decline over time after primary vaccination, but it should be remembered that the immune response to this infection does not end here. If only antibodies are quantified, only a portion of the immune response is measured.

The vaccines available against the SARS-CoV- 2 virus are based on the spike protein of the virus, focus and target of the antibodies that they will generate. It should be noted that this acquired protection-humoral immunity- after the corresponding vaccine doses, as occurs after natural infection, will be reinforced and completed by the memory immunity of $\mathrm{T}$ and $\mathrm{B}$ lymphocytes - cell-mediated immunity. By the way, this is less mentioned in the general information that is being transmitted about the definitive immune response. Antibodies decrease with time in all age groups and cellular memory although decreasing, is maintained longer [30]. Elderly individuals may have a less robust capacity to generate cellular memory due to immunosenescence and therefore be more dependent on humoral immunity.

The effectiveness achieved against infection and severe disease may decrease at the same time, but this does not necessarily occur as different immunological components come into play. Although the fall of antibodies presupposes a greater vulnerability to infection, it does not always imply a loss of immune memory and a greater predisposition to severe disease. Memory lymphocytes are guarantees for protection against reinfection and play a major role in the long-term and especially in severe disease, even when NAb titers decrease and are essential for effective vaccination $[8,29,31]$. Specifically in the normal population without known risk factors, following natural infection and in those immunized with mRNA vaccines, it has been demonstrated a robust activation and response of $\mathrm{T}$ and $\mathrm{B}$ lymphocytes in the circulation and tissues [4,32]. The genuine $T$ lymphocyte in a clonal expansion is activated in millions of copies as a defensive garrison. Another immune cell population, the memory $B$ lymphocytes like a hard disk that replenish and reactivate specific antibodies, has been shown to increase for at least six months [31], and as has happened in infections other than the present one, it could be assumed -very optimistic view?- that they are able to persist even for decades [33]. The antibody quality improves with time, becomes functionally more refined, and provides transversal protection, with far fewer antibodies needed to establish protection [34]. On the other hand, cytotoxic $\mathrm{T}$ lymphocytes eliminate already infected cells. That is, if the antibodies apparently decline in people after some time, there is a vigilant and alert cellular memory immunity, which should be the basis and fundament of vaccination.

It is necessary to be realistic and consider that in relation to the SARS-CoV-2 coronavirus and due to the time elapsed, the knowledge of the magnitude of this relationship -with the immune system-during longer periods is still limited, especially with respect to the immunity generated in vaccinated persons.

\section{Is the Cellular Memory Response Generated after Passing the Infection Similar to that after Immunization with Different Vaccines?}

Studies are already underway that may indicate the long-term behavior of these lymphocytes that make up the immunological memory. Some information is already available, such as the study by Turner JS, et al. which shows that two doses of the mRNA-based vaccine (BNT162b2) induce a persistent response of at least 15 weeks of memory $B$ cells, thus enabling a strong and reinforced humoral immunity [35]. Results of some preliminary studies suggest that this specific immunity is of better quality in convalescents. Both acquired infection and immunization with mRNA vaccines in particular, elicit an equivalent number of memory $B$ cells with rapid and robust production of antibodies in the first two months, but in those vaccinated an arrest of this antibody development was observed after two months. In those recovered from infection, memory B cells continued to progress and improve at least one year after infection had passed [32]. These findings support the need for a booster dose of vaccine. According to these authors, these differences may be related to the type of immune response that is triggered depending on whether the antigen entry point is via the upper respiratory or intramuscular route, or due to the different stimulus capacity of a whole virus or that of a spike protein. It is still too early to draw definitive conclusions until the results of further studies that are already underway are available.

The adaptive immune system is complex, raising the question of which type of immunity develops in people with acquired natural immunity and which are later vaccinated. The concurrence of both types of immunity or "hybrid immunity" is particularly of interest. A single dose of mRNA BNT162b2 vaccine in individuals with previous infection elicits a robust boosting antibody response and T-cell concentrations up to 5 times higher than in infection-naive individuals also vaccinated with a single dose [35]. Vaccination is especially potent in people who have been infected, as it generates a 25 to 100 times higher antibody response. This increase is dependent on and stimulated by T lymphocytes and memory B lymphocytes, also enabling greater protection against variants [36]. Are these individuals suitable candidates for a booster dose?

\section{Issues for Consideration}

- The long-term impact of additional doses on reducing infection, hospital admissions, and disease transmission is 
not yet clear. Although plasma antibody levels will increase and it is assumed that they may temporarily extend the protection, the magnitude to which they increase T-cell and B-cell memory responses is not yet confirmed. There is optimism that they will achieve long-term protection against severe disease for the majority of the immunocompetent population. The prediction and demonstration that antibody levels can be increased in the general population should not be interpreted as proof of long-term effectiveness, and conclusive clinical results are needed to justify the indication for these additional doses. A careful and public analysis of the course of the results obtained would appear to be opportune to ensure that decisions on extra or booster doses are based more on reliable scientific data than on political or social motives [37].

-The uncertainty of increased risk in giving additional doses in those with a history of immune-mediated reactions after the second dose of some vaccines in particular. Myocarditis for example, a very rare adverse event associated with COVID-19 mRNA vaccines has occurred at a higher incidence than in the population in pre-pandemic years. At present, its occurrence after an additional dose of mRNA vaccine is unknown [38].

-The convenience of global equity. One aspect to keep in mind is the claim that the priority should be the primary vaccination of the greatest possible number of non-immune people before even considering extra or booster vaccines in immunocompetent persons, and in all countries, especially in those with more limited resources. WHO reminds this in an appeal for global vaccine equity: "The main objective is to increase imperatively the global vaccination coverage with the primary series" [25]. Furthermore, and quite relevant, the occurrence of new and frequent infections in countries with low vaccine compliance, due to vaccine shortage (or refusal), represents a risk that higher virus replications may result in mutations and the potential emergence of resistant VOC. The survival of the virus is conditioned to the availability of a sufficient number of persons susceptible to being infected in order to replicate easily. National action is not enough; it must be global because countries that do not control the virus efficiently represent a threat to others. At the time of writing, about $52 \%$ of the world population and only $4.2 \%$ of people in poor countries have received at least 1 dose of any COVID-19 vaccine [39].

-The role of antibody-dependent enhancement phenomenon. Not all antibody responses are similar. In some viral infections, the antibodies generated in a previous infection or after vaccination can potentiate the recent secondary infection by facilitating the entry and replication of the virus (or its strains and variants) into the cells. This process is known as antibody-dependent enhancement of infection ( $A D E$ ). These are pre-existing non-neutralizing or subneutralizing antibodies, especially IgG, which recognize the antigen $[40,41]$. In short, the pre-existing antibodies become allied with the newly arrived virus, facilitating infection and potentially worsening the disease. This phenomenon has been described in infections by other viruses, such as Zika and Dengue virus and rarely after early versions of the measles vaccine [42-44]. It was speculated that a vaccine administered to persons never exposed to the virus could mimic the first infection with the production of antibodies causing that a true exposure to the natural infection of the virus would imply a more severe infectious process due to a hypothetical ADE phenomenon [43]. With regard to SARSCoV-2 infection, the virus would be able to open another lock on target cells, and also modify the transmission signal. This virus access is independent of the conventional entry pathway via the spike protein and ACE2 receptors. Results of an in vitro study show that a specific type of antibodies generated by SARS-CoV-2 infection can cause an ADE-induced infection and that these antibodies persist for at least six months after infection [45]. It has been suggested that one explanation for the emergence of the SARS-CoV-2-associated multisystemic inflammatory syndrome in children even presenting a good antibody response of SARS-CoV-2 antibodies, maybe due to the ADE phenomenon. These are non-neutralizing nonspecific anti-protein $S$ antibodies with a different activity profile [46]. There is currently no definitive clinical evidence that COVID-19 and its specific vaccines cause the ADE phenomenon. The existing data are derived basically from theoretical concepts or hypotheses or from in vitro studies.

But is ADE possible if the load and type of antibodies become very robust following the booster or extra dose in people who have already received two doses or have passed the infection?. It has been reported that in some cases of convalescent individuals, other antibodies generated may paradoxically enhance SARS-CoV-2 infection by making it easier for the spike protein to bind to ACE2, targeting specific sites in the $\mathrm{N}$-terminal domain (NTD) of this protein. $\mathrm{A}$ conformational change of the usual receptor-binding domain (RBD) occurs, which is where the infection is prevented [47]. The possible or hypothetical mechanisms of ADE in the disease could be very inherent to the virus and it must be admitted that the pathogenic components in individuals differ substantially between viruses, or even between strains of a particular virus as could be the subsequent variants of SARSCoV-2. This potential risk, although not evident in millions of people already vaccinated worldwide, should not be neglected in the prevention of this novel virus infection with the development of subsequent vaccines [48]. The vaccines have to be able to generate NAb against potential VOC with an adequate immune response. If this is not the case, the generated non-neutralizing antibodies would facilitate the ADE phenomenon [40].

\section{Conclusions}

- According to some experts opinion, the currently available information does not seem to show a strong need for a booster dose to the general immunocompetent population given that the vaccine effectiveness against severe disease is high and persistent, being more justified especially if the interest is to better prevent infection [32-34,36,37]. However, some countries are already recommending the administration of additional doses of vaccines in immunocompetent persons without other risk factors, not elderly, even as early as 18 years of age [26]. 
Citation: Álvez F (2022) Additional Doses of Vaccines against COVID-19 - Attempting to Enhance the Immune Response. Vaccine Res Dev $3(1): 9-15$

- From now on, the introduction of additional doses of vaccines and their composition should be firmly based on clinical and observational studies and directed to the population groups most in need. Evidence showing reduced effectiveness of vaccines, in particular in protection against severe disease in the general population and in high-risk populations, or due to some circulating VOC. At present, the available information is still limited and inconclusive as to the systematic need for additional doses after a primary vaccination series [25]. Regarding the duration of immunity, there is more data available on mRNA vaccines than on other vaccines with different characteristics, namely protein-based vaccines [49].

-While accepting the imminence of an extra or booster dose, the uncertainty with the emergence of more VOC capable of partially evading the immune response to current vaccines based on the original strain of the virus, compels and justifies the search for "broad-spectrum" or universal vaccines. They should be of new generation against SARSCoV-2 and new coronaviruses, protecting against infection with all known and emerging VOC. Studies are already underway to develop vaccines of this type. Ideally, one hopes for a vaccine that would cover not only SARS-CoV-2 and its variants, but other coronaviruses with known potential to cause severe disease in people in the future, most likely from the subgenus Sarbecovirus to which SARS-CoV-2 belongs. In other words, a universal pan-sarbecovirus vaccine $[50,51]$. Both systemic and nasal mucosal immunity are equally important. The vaccines now available, all administered intramuscularly, generate circulating antibodies in the blood, but with a slower response at the level of the nasal mucosa, a recognized gateway for the virus. The Delta and Omicron variants replicates much more in the respiratory tract than other variants. Intranasal vaccines under development have the potential to greatly reduce respiratory transmission and are expected to able to induce a strong immune response similar to that achieved by the intramuscular route [52].

-As things stand and taking into account the threat of other VOC with potential transmission capacity, and the concern about antigenic changes that may condition vaccine protection, the prediction is that it will not be possible to eliminate SARS-CoV-2, and the population will have to adapt, confront it and learn to live with this virus. We are aware of a scenario in which vaccinated persons may become infected and present mild and asymptomatic processes if more VOC continue to appear and, the more remote possibility that the disease will be severe in a small proportion of vaccinated persons. In general, the situation will be changing due to the behavior of the virus and the idiosyncrasy of the population, but it will be imperative to continue vaccinating to avoid serious consequences. The presence of antibodies plays an important role in the prevention of severe disease, hospitalization, and death in the event of reinfection or breakthrough infections. The course of time will show the true preventive effectiveness of the additional doses of the vaccines and whether or not they need to be repeated periodically, annually, as is the case with the influenza virus. In the meantime, let the search continue for antiviral therapeutic agents that will not be conditioned by the molecular changes of the viruses.

\section{Declarations}

- Ethics approval and consent to participate: No applicable.

- Consent for publication: No applicable.

- Availability of data and materials: No applicable.

- Funding and competing interest: No funding was provided for this manuscript. Author declares no conflict of interest.

- Authors' contributions: No applicable.

- Acknowledgements: No applicable.

\section{References}

1. Beaudoin-Bussières G, Laumaea A, Anand SP, et al. (2020) Decline of humoral responses against SARS-CoV-2 Spike in convalescent individuals. mBio 11: e02590-20.

2. Khoury DS, Cromer D, Reynaldi A, et al. (2021) Neutralizing antibody levels are highly predictive of immune protection from symptomatic SARS-CoV-2 infection. Nat Med 27: 1205-1211.

3. Wang K, Long QL, Deng HJ, et al. (2021) Longitudinal dynamics of the neutralizing antibody response to severe acute respiratory syndrome coronavirus 2 (SARS-CoV-2) infection. Clin Infect Dis 73: e531-e539.

4. Zhang J, Lin $\mathrm{H}, \mathrm{Ye} \mathrm{B}$, et al. (2021) One-year sustained cellular and humoral immunities of COVID-19 convalescents, Clin Infect Dis ciab884.

5. Haveri A, Ekström N, Solastie A, et al. (2021) Persistence of neutralizing antibodies a year after SARS-CoV-2 infection. Eur J Immunol 51: 3203-3213.

6. Choe PG, Kang KCh, Kim KH, et al. (2021) Persistence of neutralizing antibody response up to 1 year after asymptomatic or symptomatic SARS-CoV-2 Infection. J Infect Dis 224: 1097-1099.

7. Tregoning JS, Flight KE, Higham SL, et al. (2021) Progress of the COVID-19 vaccine effort: viruses, vaccines and variants versus efficacy, effectiveness and escape. Nat Rev Immunol 21: 626-636.

8. Cevik M, Grubaugh ND, Iwasaki A, et al. (2021) COVID-19 vaccines: Keeping pace with SARS-CoV-2 variants. Cell 184: 5077-5081.

9. Levin EG, Lustig Y, Cohen C et al. (2021) Waning immune humoral response to BNT162b2 Covid-19 vaccine over 6 months. N Engl J Med 385: e84.

10. Chemaitelly $H$, Tang $P$, Hasan MR, et al. (2021) Waning of BNT162b2 vaccine protection against SARS-CoV-2 infection in Qatar. N Engl J Med 385: e83.

11. Tenforde MW, Self WH, Adams K, et al. (2021) Association between mRNA Vaccination and COVID-19 Hospitalization and Disease Severity. JAMA 326: 2043-2054.

12. Self WH, Tenforde MW, Rhoads JP, et al. (2021) Comparative effectiveness of moderna, Pfizer-BioNTech, 
Citation: Álvez F (2022) Additional Doses of Vaccines against COVID-19 - Attempting to Enhance the Immune Response. Vaccine Res Dev $3(1): 9-15$

and Janssen (Johnson \& Johnson) vaccines in preventing COVID-19 hospitalizations among adults without immunocompromising conditions - United States, MarchAugust 2021. MMWR Morb Mortal Wkly Rep 70: 13371343.

13. Sheikh A, Robertson C, Taylor B (2021) BNT162b2 and ChAdOx1 nCoV-19 Vaccine Effectiveness against death from the delta variant. N Engl J Med 385: 2195-2197.

14. Reis BY, Barda N, Leshchinsky M, et al. (2021) Effectiveness of BNT162b2 vaccine against delta variant in adolescents. N Engl J Med 385: 2101-2103.

15. Dagan N, Barda N, Kepten E, et al. (2021) BNT162b2 mRNA Covid-19 vaccine in a nationwide mass vaccination setting. N Engl J Med 384: 1412-1423.

16. Nordström P, Ballin M, Nordström A (2021) Association between risk of COVID-19 infection in nonimmune Individuals and COVID-19 immunity in their family members. JAMA Intern Med 181: 1589-1595.

17. Shah ASV, Gribben C, Bishop J, et al. (2021) Effect of vaccination on transmission of SARS-CoV-2. N Engl J Med 385: 1718-1720.

18. Levine-Tiefenbrun M, Yelin I, Katz R, et al. (2021) Initial report of decreased SARS-CoV-2 viral load after inoculation with the BNT162b2 vaccine. Nat Med 27: 790-792.

19. Thompson MG, Burgess JL, Naleway AL, et al. (2021) Prevention and attenuation of Covid-19 with the BNT162b2 and mRNA-1273 vaccines. N Engl J Med 385: 320-329.

20. Singanayagam A, Hakki S, Dunning J, et al. (2021) Community transmission and viral load kinetics of the SARS-CoV-2 delta (B.1.617.2) variant in vaccinated and unvaccinated individuals in the UK: A prospective, longitudinal, cohort study. Lancet Infect Dis 34756187.

21. Tartof SY, Slezak JM, Fischer H, et al. (2021) Effectiveness of mRNA \&BNT162b2 COVID-19 vaccine up to 6 months in a large integrated health system in the USA: A retrospective cohort study. Lancet 398: 1407-1416.

22. Andrews N, Tessier E, Stowe J, et al. (2021) Vaccine effectiveness and duration of protection of Comirnaty, Vaxzevria and Spikevax against mild and severe COVID-19 in the UK. medRxiv.

23. Scott J, Richterman A, Cevik M, et al. (2021) Covid-19 vaccination: evidence of waning immunity is overstated. BMJ 374: n2320.

24. Rosenberg ES, Holtgrave DR, Dorabawila V, et al. (2021) New COVID-19 Cases and hospitalizations among adults, by vaccination Status - New York, May 3-July 25, 2021. MMWR Morb Mortal Wkly Rep 70: 1306-1311.

25. WHO (2021) Interim statement on booster doses for COVID-19 vaccination.

26. Centers for Disease Control and Prevention (CDC) (2021) COVID-19 vaccine booster dose.
27. FDA Press Announcements (2021) Coronavirus (COVID-19) Update: FDA Takes Additional Actions on the Use of a Booster Dose for COVID-19 Vaccines.

28. Mbaeyi S, Oliver SE, Collins JP, et al. (2021) The Advisory Committee on Immunization Practices' Interim Recommendations for Additional Primary and Booster Doses of COVID-19 Vaccines United States, 2021. MMWR Morb Mortal Wkly Rep 70: 1545-1552.

29. European Centre for Disease Prevention and Control (2021) Interim public health considerations for the provision of additional COVID-19 vaccine doses.

30. Stephens DS, Mc Elrath MJ (2020) COVID-19 and the path to immunity. JAMA 324: 1279-1281.

31. Gaebler C, Wang Z, Lorenzi, et al. (2021) Evolution of antibody immunity to SARS-CoV-2. Nature 591: 639-644.

32. Cho A, Muecksch F, Dennis SB, et al. (2021) Anti-SARSCoV-2 receptor binding domain antibody evolution after mRNA vaccination. Nature 600: 517-522.

33. Amanna IJ, Carlson NE, Slifka MK (2007) Duration of humoral immunity to common viral and vaccine antigens. N Engl J Med 357: 1903-1915.

34. Turner JS, O'Halloran JA, Kalaidina E, et al. (2021) SARSCoV-2 mRNA vaccines induce persistent human germinal centre responses. Nature 596: 109-113.

35. Angyal A, Longet S, Moore SC, et al. (2021) T-cell and antibody responses to first BNT162b2 vaccine dose in previously infected and SARS-CoV-2-naive UK health-care workers: a multicentre prospective cohort study. Lancet Microbe 3: e21-e31.

36. Crotty S (2021) Hybrid immunity. Science 372: 1392-1393.

37. Krause PR, Fleming TR, Peto R, et al. (2021) Considerations in boosting COVID-19 vaccine immune responses. Lancet 398: 1377-1380.

38. Perez Y, Levy ER, Joshi AY, et al. (2021) Myocarditis following COVID-19 mRNA vaccine: A case series and incidence rate determination. Clin Infect Dis ciab926.

39. Ritchie H, Mathieu E, Rodés-Guirao L, et al. (2021) What share of the population has been partly or fully vaccinated against COVID-19? Our World in Data.

40. Sánchez-Zuno GA, Matuz-Flores MG, González-Estevez G, et al. (2021) A review: Antibody-dependent enhancement in COVID-19: The not so friendly side of antibodies. Int J Immunopathol Pharmacol 35: 1-15.

41. Bastug A, Bodur H (2022) SARS-CoV-2 Infection and Antibody-Dependent Enhancement. Studies in Computational Intelligence 963: 101-113.

42. Rothan HA, Bidokhti MRM, Byrareddy SN (2018) Current concerns and perspectives on Zika virus co-infection with arboviruses and HIV. J Autoimmun 89: 11-20.

43. Sridhar S, Luedtke A, Langevin E, et al. (2018) Effect of dengue serostatus on dengue vaccine safety and efficacy. N Engl J Med 379: 327-340. 
44. Martin DB, Weiner LB, Nieburg PI, et al. (1979) Atypical measles in adolescents and young adults. Ann Intern Med 90: 877-881.

45. Maemura T, Kuroda M, Armbrust T, et al. (2021) Antibodydependent enhancement of SARS-CoV-2 infection is mediated by the IgG receptors FcyRIIA and FcyRIIIA but does not contribute to aberrant cytokine production by macrophages. mBio 12: 0198721.

46. Rothan HA, Byraddedy SN (2021) The potential threat of multisystem inflammatory syndrome in children during the COVID-19 pandemic. Pediatr Allergy Inmunol 32: 17-22.

47. Liu Y, Soh WT, Kishikawa Jl, et al. (2021) An infectivityenhancing site on the SARS-CoV-2 spike protein targeted by antibodies. Cell 184: 3452-3466.
48. Arvin AM, Fink K, Schmid MA, et al. (2020) A perspective on potential antibody-dependent enhancement of SARSCoV-2. Nature 584: 353-363.

49. Monto A (2021) The Future of SARS-CoV-2 Vaccination Lessons from Influenza. N Engl J Med 385: 1825-1827.

50. Li H, Saphire EO (2021) Novel attempts launched toward universal Sarbecovirus vaccine. Cell Res 31: 1226-1227.

51. Tan CW, Chia WN, Young BE, et al. (2021) Pan-Sarbecovirus neutralizing antibodies in BNT162b2-Immunized SARSCoV-1 survivors. N Engl J Med 385: 1401-1406.

52. Rubin R (2021) Trying to block SARS-CoV-2 transmission with intranasal vaccines. JAMA 326: 1661-1663.

DOI: $10.36959 / 669 / 748$ 